\title{
FERTILIDADE DE GEMAS DE VIDEIRAS 'NIAGARA ROSADA' DE ACORDO COM O SISTEMA DE CONDUÇÃO ${ }^{1}$
}

\author{
CÁSSIA REGINA YURIKO IDE VIEIRA², ERASMO JOSÉ PAIOLI PIRES ${ }^{3}$, MARCO ANTONIO TECCHIO4, \\ ILDA MIYUKI NAKASE OTSUBO ${ }^{5}$, MARIA DO CARMO VIEIRA ${ }^{6}$, ALBERTO KAZUMI YAMASAKI ${ }^{5}$, \\ OTÍLIA BORTOLANZA
}

\begin{abstract}
RESUMO - O experimento teve por objetivo avaliar a influência dos sistemas de condução espaldeira e pérgula, na fertilidade das gemas da videira 'Niagara Rosada' na Região de Dourados-MS, durante o período de maio a junho de 2004. Coletaram-se bacelos provenientes de 24 vinhedos de plantas da cultivar Niagara Rosada enxertadas sobre o porta-enxerto IAC 572 'Jales', conduzidas em espaldeira e pérgula, que se encontravam na fase produtiva. Foram realizadas as seguintes avaliações: número de primórdios de inflorescência/gema primária (NPI/GP), número total de gemas/ gema composta (NTG/GC) e número de gemas necrosadas (NGN). O delineamento foi em blocos casualizados, com 12 repetições, no esquema fatorial 2 x 10, usando-se como fatores dois sistemas de condução, em espaldeira e em pérgula; e dez posições da gema na vara, isto é, da basal à décima gema. Os blocos consistiram de 12 vinhedos de plantas conduzidas em espaldeira e outros 12 em pérgula. As médias dos dados foram comparadas pelo Teste de Tukey, a 5\% de probabilidade. Verificou-se que, nas videiras conduzidas em pérgula, as médias de fertilidade de gemas são maiores que as do sistema em espaldeira, e a fertilidade de gemas é crescente da base à porção terminal das varas, nos dois sistemas de condução analisados.
\end{abstract}

Termos para indexação: Vitis, Niagara Rosada, fertilidade de gemas.

\section{BUD FERTILITY OF GRAPEVINES 'NIAGARA ROSADA' IN ACCORDANCE WITH THE CONDUCTION SYSTEM}

ABSTRACT - The objective of this trial was to evaluate the influence of the cordon training and trellis, on bud fertility of the grapevine Niagara Rosada in the Region of Dourados, MS, during the period from May to June, 2004. The shoots were collected from 24 vineyards conducted on cordon-training or trellis. The bud fertility index was determined by the following ratios: number of primordia of inflorescence/primary buds, and the total number of buds/compound buds, and by the number of necrosed buds, from the first to the tenth dormant buds on the mature vine cane. The experimental design was complete randomized blocks with 12 repetitions, in a factorial $2 \times 10$. The factors studied were the cordon training and the trellis, and the bud position along the cane. The blocks consisted of 12 grapevines conduced on cordon-training and 12 conduced on trellis. The experimental unity consisted of 10 canes. Means were compared by the Tukey test at 5\%. It has been concluded that grapevines conducted on the trellis presented higher averages on bud fertility when compared with cordon-training. The bud fertility presented increasing averages from the basis to the terminal portion of the shoot, in both training systems.

Index terms: Vitis, Niagara Rosada, bud fertility.

No Mato Grosso do Sul, a viticultura encontra-se em expansão. No entanto, a produtividade e a qualidade das uvas não são satisfatórias, sendo necessárias mais pesquisas visando à melhoria do sistema de produção adotado, para a viabilização da cultura na região. Reduzidas produtividades e qualidade de frutos podem estar associadas, dentre outros fatores, aos sistemas de condução adotados que, na região, geralmente são conduzidas as videiras em espaldeira com duplo cordão esporonado ou pérgula.

A fertilidade das gemas é a capacidade das mesmas de diferenciar gemas vegetativas em florais, podendo ser utilizada para estimativa quantitativa do potencial produtivo de frutos de um determinado vinhedo. De acordo com Srinivasan \& Mullins (1981), essa diferenciação ocorre em três estádios bem definidos: formação do blastema ou primórdio não comprometido, diferenciação do blastema para a formação dos primórdios de inflorescência ou primórdios de gavinha e formação de flores. Os dois primeiros estádios ocorrem durante a fase de crescimento dos ramos do ciclo anterior, e dura aproximadamente 45 a 60 dias, a partir do início da brotação. O estádio final, da formação de flores, ocorre um pouco antes e durante a brotação do ciclo seguinte.

Qualquer desbalanço entre os fatores responsáveis para a formação de primórdios de inflorescência poderá levar o blastema a diferenciar-se em gavinha ou broto vegetativo. A formação de gema frutífera é influenciada pela luz, citocinina, ácido ribonucleico (Shikhamany,1999), temperatura, característica varietal, vigor dos ramos, déficit hídrico, desponte, desnetamento ou sistema de condução, dentre outros fatores (Haggag et al., 1996; Shikhamany,1999; Hepner et al., 1985; Bravdo \& Naor, 1996; Mullins et al., 1992; Sommer et al., 2000).

O conhecimento da posição das gemas férteis em cada variedade é uma importante informação para a orientação do tipo de poda a ser empregado no vinhedo, com conseqüentes aumentos em produtividade. Sansavini \& Fanigulo (1998) observaram, em experimento realizado em Metaponto, na Itália, que videiras 'Centennial Seedless' e 'Sugraone' apresentaram maior fertilidade entre a $6^{\mathrm{a}}$ e a $18^{\mathrm{a}}$ gemas, com maiores produtividades em plantas submetidas às podas longas. As plantas que receberam as podas médias e curtas apresentaram menor tamanho das bagas e dos cachos, e o teor de sólidos solúveis totais do mosto, e a produtividade foi drasticamente reduzida.

A redução da fertilidade também pode ser devida à necrose de gemas das videiras, que é uma desordem fisiológica, sendo normalmente abortadas as gemas primárias e, ocasionalmente, as secundárias. Está associada ao alto vigor e diâmetro de ramos (Dry \& Coombe, 1994; Lavee, 1987; Wolf e Warren, 1995), baixo nível de carboidrato em tecidos

\footnotetext{
(Trabalho 184/2005). Recebido: 09/11/2005. Aceito para publicação: 08/03/2006.

2 Eng. Agr., Dra, Pesquisadora, Idaterra/MS, Caixa Postal 17, 79 803-040, Dourados, MS. Telefones: (67)422-2128, (67) 99283728. Endereço: cassiar@fca.unesp.br. Eng. Agr, Dr, Pesquisador, Instituto Agronômico de Campinas, Bolsista do CNPq, Caixa Postal, 28. 13.001-970, Campinas - SP. Telefone: (19) 3241-9910. Endereço: ejppires@iac.sp.gov.br.

${ }^{4}$ Eng. Agr, Dr, Pesquisador, Instituto Agronômico de Campinas. Av. Luiz Pereira dos Santos, 1500, Bairro Corrupira - 13214-820. Jundiaí - SP. Telefone: (11) 4582-7284. Endereço: tecchio@iac.sp.gov.br.

5 Acadêmico(a) do Curso de Graduação em Agronomia da Faculdade de Agronomia de Dourados - FAD/UNIDERP. Av. Presidente Vargas, 1775, Vila Progresso, CEP 79.825-090. Dourados-MS. Telefone: (67) 411-1800. Endereços: amy bibi@hotmail.com; michelibortolanza@hotmail.com; alberto yamasaki@yahoo.com.br. ${ }^{6}$ Eng. Agr. Dra. Profa. titular Departamento de Ciências Agronômicas, UFMS, Caixa Postal 533, CEP 79.804-970. Dourados - MS, telefone (67) 411-3845. Endereço: vieiracm@terra.com.br.
} 
TABELA 1 - Primórdios de inflorescência, gemas necrosadas por gema composta e total de gemas, de varas de videiras 'Niagara Rosada' conduzidas em sistemas de espaldeira e pérgula. Dourados-MS, 2004.

\begin{tabular}{|c|c|c|c|}
\hline Sistema de condução & Primórdios de Inflorescência/gema primária & Número de Gemas necrosadas & $\mathrm{N}^{0}$ total de gemas / gema composta \\
\hline Espaldeira & $1.32 \mathrm{~b}$ & $0,81 \mathrm{a}$ & $1,56 \mathrm{a}$ \\
\hline Pérgula & $1.57 \mathrm{a}$ & $0,78 \mathrm{~b}$ & $1,57 \mathrm{a}$ \\
\hline
\end{tabular}

Médias seguidas pela mesma letra na coluna não diferem entre si, pelo Teste de Tukey, ao nível de 5\% de probabilidade.

de gema (Naito et al. ,1987), sombreamento de gemas (Pérez e Kliewer, 1990, Wolf e Warren, 1995) e alta atividade de giberelina nas gemas (Lavee, 1987). Irrigação excessiva e altos níveis de giberelina aumentam o vigor dos ramos, os quais, sob certas condições, podem resultar em carreamento de carboidratos para outros órgãos. Sombreamento também tem sido relatado por reduzir fotossíntese da planta e conseqüente diminuição dos teores de carboidrato (Cartechini e Palliotti,1995). Brotação menos vigorosa aumenta a penetração de luz nas gemas (Pérez e Kliewer, 1990), diminuindo a incidência de gemas necróticas. As causas para essa necrose não estão totalmente esclarecidas, mas o vigor de ramos e sombreamento de gemas têm sido relatados como as principais (Edson et al.,1995).

A condução da videira pode também influenciar na incidência de luz solar nas gemas. Em videiras conduzidas em sistema "Y", podem resultar copas abertas, com ramos orientados em diagonal, permitindose maior incidência de luz nas gemas, resultando em aumentos na produtividade de frutos (Shikhamany, 1999).

No presente trabalho, teve-se como objetivo avaliar a fertilidade de gemas de videiras de 'Niagara Rosada' na região de Dourados-MS, em função dos sistemas de condução em espaldeira e pérgula.

O experimento foi desenvolvido no Laboratório de Botânica da FAD/UNIDERP, em Dourados - MS, de maio a junho de 2004 utilizando-se de gemas coletadas em plantas de 24 vinhedos de 'Niagara Rosada' enxertadas sobre o porta-enxerto IAC 572 'Jales', conduzidas em sistema de espaldeira e de pérgula, na fase produtiva, nos municípios de Itaporã, Maracaju, Dourados, Fátima do Sul, Rio Brilhante e Ponta Porá-MS. O material amostrado foi coletado em plantas podadas no ciclo anterior, em agosto de 2003.

O sistema de espaldeira com duplo cordão esporonado adotado pelos produtores da região é composto por cinco fios, sendo que os dois primeiros ficam a $1 \mathrm{~m}$ do solo e estes distanciados entre si por um espaçador de madeira de $60 \mathrm{~cm}$, o segundo encontra-se a 1,30 m do solo e distanciados entre si por um espaçador de madeira de 40 a 50 $\mathrm{cm}$ e o terceiro a $1,70 \mathrm{~m}$ do solo. As plantas conduzidas neste sistema formam um túnel, ficando a parte interna sombreada.

O índice de fertilidade da primeira à décima gema da vara foi determinado com o auxílio de uma lupa estereoscópica, calculando-se o número de primórdios de inflorescência/gema primária (NPI/GP), o número total de gemas/gema composta (NTG/GC) e o número de gemas necrosadas (NGN).

O delineamento foi em blocos casualizados, com 12 repetições, no esquema fatorial 2 × 10 , usando-se como fatores dois sistemas de condução, espaldeira e pérgula, e 10 posições da gema na vara, sendo P1 a gema basal e P10 a gema apical. A unidade experimental consistiu da média de 10 varas. Os dados coletados foram submetidos à análise de variância e as médias comparadas pelo teste de Tukey, ao nível de $5 \%$ de probabilidade. Os valores das variáveis estudadas foram transformados em $(x+0,5)^{0,5}$.

O sistema espaldeira com duplo cordão esporonado promoveu os menores índices de fertilidade e maior NGN (Tabela 1). Tais fatos podem estar associados à menor incidência de luz nas gemas neste sistema de condução, visto que a parte interna das plantas fica sombreada e à dominância apical, que é maior em ramos conduzidos verticalmente (Shikhamany, 1999). Nestas condições, tem-se alto nível de auxinas no meristena apical (Taiz \& Zeiger, 1991), o que resulta em inibição da formação de conexões vasculares e restrição de nutrientes (Taiz \& Zeiger, 1991). As citocininas estimulam o crescimento das gemas laterais e mobilizam assimilados. A síntese de citocininas nas gemas laterais é inibida pelas auxinas do ápice e da própria gema (Metivier, 1979). Portanto, removendo-se as gemas apicais, ou melhor, a dominância apical, o nível de citocininas, de nutrientes ou de ambos, em gemas laterais, se eleva, promovendo a maior fertilidade das gemas (Taiz \& Zeiger, 1991; Naito et al., 1987; Shikhamany, 1999).

Nos dois sistemas de condução considerados, as maiores médias de fertilidade de gemas e os menores números de gemas necrosadas foram verificados na porção terminal das varas (Tabela 2), o que pode ser em conseqüência de maior acúmulo de carboidratos nessa região (Winkler, 1965). De acordo com os resultados, constata-se a necessidade de reformulação do sistema de produção adotado para a cultura para se obter maior produtividade nos vinhedos. Em caso de videiras conduzidas em sistema de pérgula, normalmente, faz-se a poda, deixando-se 2 gemas por vara podada. No entanto, deixando-se 4 gemas por vara podada, com quebra da dormência das duas últimas, pode-se ter um acréscimo de $20 \%$ de primórdios de inflorescência, o que poderá resultar em maiores produtividades. Para a variável número total de gemas/gema composta, não houve diferenças significativas entre os dois sistemas de condução e nas diferentes posições das gemas nas varas (Tabelas 1 e 2). De acordo com os resultados obtidos neste trabalho, conclui-se que:

1. A fertilidade das gemas é maior em videiras conduzidas em sistema de pérgula do que em espaldeira.

2. A fertilidade de gemas é crescente da base à porção terminal das varas, nos sistemas de condução em espaldeira e em pérgula.

TABELA 2 - Primórdios de inflorescência/gema primária, gemas necrosadas/gema composta e número total de gemas/gema composta de varas de videiras 'Niagara Rosada' conduzidas em sistema de espaldeira e pérgula. Dourados-MS, 2004.

\begin{tabular}{lccc}
\hline $\begin{array}{c}\text { Posição das gemas nas } \\
\text { varas }\end{array}$ & $\begin{array}{c}\text { Primórdios de Inflorescência / gema } \\
\text { primária }\end{array}$ & $\begin{array}{c}\text { Número de Gemas necrosadas / gema } \\
\text { composta }\end{array}$ & $\begin{array}{c}\mathrm{N}^{0} \text { total de gemas / gema } \\
\text { composta }\end{array}$ \\
\hline 1 & $1,17 \mathrm{~b}$ & $0,85 \mathrm{a}$ & $1,54 \mathrm{a}$ \\
2 & $1,19 \mathrm{~b}$ & $0,83 \mathrm{ab}$ & $1,56 \mathrm{a}$ \\
3 & $1,36 \mathrm{ab}$ & $0,83 \mathrm{ab}$ & $1,55 \mathrm{a}$ \\
4 & $1,53 \mathrm{a}$ & $0,79 \mathrm{abc}$ & $1,57 \mathrm{a}$ \\
5 & $1,48 \mathrm{a}$ & $0,81 \mathrm{abc}$ & $1,58 \mathrm{a}$ \\
6 & $1,57 \mathrm{a}$ & $0,78 \mathrm{abc}$ & $1,57 \mathrm{a}$ \\
7 & $1,60 \mathrm{a}$ & $0,77 \mathrm{bc}$ & $1,58 \mathrm{a}$ \\
8 & $1,56 \mathrm{a}$ & $0,77 \mathrm{bc}$ & $1,58 \mathrm{a}$ \\
9 & $1,50 \mathrm{a}$ & $0,75 \mathrm{c}$ & $1,57 \mathrm{a}$ \\
10 & $1,47 \mathrm{a}$ & $0,77 \mathrm{bc}$ & $1,58 \mathrm{a}$ \\
\hline $\mathrm{CV}(\%)$ & 8,018 & 9,83 & 2,37 \\
\hline
\end{tabular}

Médias seguidas pela mesma letra na coluna não diferem entre si, pelo Teste de Tukey, ao nível de $5 \%$ de probabilidade. 


\section{REFERÊNCIAS}

BRAVDO, B.; NAOR, A. Effect of water regime on productity and quality of fruit and wine. Acta Horticulturae, Leiden, v.427, p.15-26, 1996.

CARTECHINI, A.; PALLIOTTI, A. Effect of shading on vine morphology and productivity and leaf gas exchange characteristics in grapevines in the field. American Journal of Enology and Viticulture, Davis, v.41, n.2, 1995.

DRY, P. R.; COOMBE, B. G. Primary bud-axis necrosis of grapevines. I. Natural incidence and correlation with vigor. Vitis, Siebelding, v. 33, p. 225-230. 1994.

EDSON, C. E.; HOWELL, G. S.; FLORE, J. A. Influence of crop load on photosynthesis and dry matter partitioning of Seyval grapevines. II. Seasonal changes in single leaf and whole vine photosynthesis. American Journal of Enology amd Viticulture, Davis, 46, p. 469485, 1995.

HAGGAG, M.N.; ETMAN, A.A.; EL SHAZLY, S.M.; EL SHARKAWY, I.G. Comparative studies and evaluation of fourteen cultivars in Egypt. I. Bud fertility, Bud fertility coefficient, yield, nd fruit qulity, and the morphological characteristics. Alexandria Journal of Agricultural Research, Alexandria, v.41, n.3, p.275-291, 1996.

HEPNER, Y.; BRAVDO, B.; LOINGER, S.; COHEN, S.; TABACMAN,A. Effect of drip irrigation schedules on growth, yield, composition and winw quality of Cabernet Sauvignon. American Journal of Enology and Viticulture, Davis, v.36, p.77-85, 1985.

LAVEE, S. Necrosis in grapevine buds (Vitis vinifera cv. Queen of Vineyard). III. endogenous gibberellin levels in leaves and buds. Vitis, Davis, v. 26, p. 225-230, 1987.

METIVIER, J.R. Citocininas. In: FERRI, M.G. (Coord). Fisiologia vegetal 2. São Paulo: EPU./EDUSP, 1979. p. 93-127.

MULLINS, M.G.; BOUQUET, A.; WILLIANS, L.E. Developmental physiology: flowering and fuiting. In: MULLINS, M.G. (Ed). Biology of the grapevine. Cambridge: University Press, 1992. p.112-146.
NAITO, R.; YAMAMURA, H.; MUNESUE, S. Studies on the necrosis in grapevine buds (III) the time of the occurrence of bud necrosis in 'Kyoho' and the relation between its occurrence and the amounts of nutritional elements in buds. Bull. Fac. Agric. Shimane University NAITO, R.; YAMAMURA, H.; MUNESUE, S. Studies on the necrosis in grapevine buds (III) the time of the occurrence of bud necrosis Bulletin Faculty of Agriculture Shimane University, Nogakubu, v. 21, p.10-17, 1987.

PÉREZ, J. AND KLIEWER, W. M. Effect of shading on bud necrosis and bud fruitfulness of 'Thompson Seedless' grapevines. American Journal of Enology and Viticulture, Davis, v.41, p. 168$175,1990$.

SANSAVINI, S.; FANIGLIUGO, G. Fertilita delle gemme e influenza della potatura sulla fruttifica zione delle uve pirene "Centennial Seedless" e " "Sugreone". Rivista di Frutticoltura e di Ortofloricoltura", Itália, v.60, n.2, p. 55-60, 1998.

SKIKHAMANY, S.D. Physiology and cultural practices to produce eedless grapes in tropical environments. In: CONGRESSO BRASILEIRO DE VITICULTURA E ENOLOGIA, 9., 1999. Bento Gonçalves. Anais... Bento Gonçalves: Embrapa-CNPUV, p.43-48, 1999.

SOMMER, K. J.; ISLAM, M. T.; CLINGELEFFER, P. R. Light and temperature effects on shoot fruitfulness in Vitis vinifera L. cv. Sultana: influence of trellis type and grafting. Australian Journal of Grape and Wine Research, Austrália, n.6, p.99-108, 2000.

SRINIVASAN, C.; MULLINS, M.G. Phyiology of flowering in the grapevine. American Journal of Enology and viticulture, Davis, v.32, n.1, p.47-63, 1981.

TAIZ, L.; ZEIGER, E. Plant physiology. Califórnia: The Benjamin/ Cummings Publishings Company, 1991.565p.

WINKLER, A. J. Viticultura. México:Continental, 1965. 792p.

WOLF, T. K.; WARREN, M. K. Shoot growth rate and shoot density affect bud necrosis of 'Riesling' grapevines. Journal of the American Society for Horticultural Science, Alexandria, v.120, p. 989-996,1995. 